\title{
CLASSICAL PROPOSITIONAL LOGIC AND DECIDABILITY OF VARIABLES IN INTUITIONISTIC PROPOSITIONAL LOGIC
}

\author{
HAJIME ISHIHARA
}

School of Information Science, Japan Advanced Institute of Science and Technology, Nomi, Ishikawa 923-1292, Japan

e-mail address: ishihara@jaist.ac.jp

\begin{abstract}
We improve the answer to the question: what set of excluded middles for propositional variables in a formula suffices to prove the formula in intuitionistic propositional logic whenever it is provable in classical propositional logic.
\end{abstract}

\section{INTRODUCTION}

Let $\vdash_{c}$ and $\vdash_{i}$ denote derivability in classical and intuitionistic propositional logic, respectively. Then it is known that if $\vdash_{c} A$, then $\Pi_{\mathcal{V}(A)} \vdash_{i} A$, where $\mathcal{V}(A)$ is the set of propositional variables in a formula $A$ and $\Pi_{V}=\{p \vee \neg p \mid p \in V\}$ for a set $V$ of propositional variables; see, for example, [1, appendix], and [4, p. 27] which was originally given in [7].

In this note, we consider a problem: what set $V$ of propositional variables suffices for $\Pi_{V}, \Gamma \vdash_{i} A$ whenever $\Gamma \vdash_{c} A$, and show, employing a technique in [2, 3], that $V=\left(\mathcal{V}^{-}(\Gamma) \cup\right.$ $\left.\mathcal{V}^{+}(A)\right) \cap\left(\mathcal{V}_{n s}^{+}(\Gamma) \cup \mathcal{V}^{-}(A)\right)$ suffices, where $\mathcal{V}^{+}, \mathcal{V}^{-}$and $\mathcal{V}_{n s}^{+}$are the sets of propositional variables occurring positively, negatively and non-strictly positively, respectively (precise definitions will be given in the next section). For example, since $(p \rightarrow q) \rightarrow p \vdash_{c} p$, we have

$$
p \vee \neg p,(p \rightarrow q) \rightarrow p \vdash_{i} p
$$

and, since $p \rightarrow q \vee r \vdash_{c}(p \rightarrow q) \vee(p \rightarrow r)$, we have

$$
p \vee \neg p, p \rightarrow q \vee r \vdash_{i}(p \rightarrow q) \vee(p \rightarrow r) .
$$

2012 ACM CCS: [Theory of computation]: Logic.

Key words and phrases: classical propositional logic, intuitionistic propositional logic, decidability of variables . 


\section{Preliminaries}

We refer to Troelstra and Schwichtenberg [6] for the necessary background on sequent calculi; see also Negri and von Plato [4. We use the standard language of propositional logic containing $\wedge, \vee, \rightarrow$ and $\perp$ as primitive logical operators, and introduce the abbreviation $\neg A \equiv A \rightarrow \perp$. We define positive, strictly positive and negative occurrence of a formula in the usual way (see [6, 1.1.3] or [5, 3.9,3.11,3.23] for details). The sets $\mathcal{V}^{+}(A)$ and $\mathcal{V}^{-}(A)$ of propositional variables occurring positively and negatively, respectively, in a formula $A$ are simultaneously defined by

$$
\begin{aligned}
\mathcal{V}^{+}(p) & =\{p\}, \quad \mathcal{V}^{+}(\perp)=\emptyset \\
\mathcal{V}^{+}(A \wedge B) & =\mathcal{V}^{+}(A \vee B)=\mathcal{V}^{+}(A) \cup \mathcal{V}^{+}(B), \\
\mathcal{V}^{+}(A \rightarrow B) & =\mathcal{V}^{-}(A) \cup \mathcal{V}^{+}(B), \\
\mathcal{V}^{-}(p) & =\mathcal{V}^{-}(\perp)=\emptyset, \\
\mathcal{V}^{-}(A \wedge B) & =\mathcal{V}^{-}(A \vee B)=\mathcal{V}^{-}(A) \cup \mathcal{V}^{-}(B), \\
\mathcal{V}^{-}(A \rightarrow B) & =\mathcal{V}^{+}(A) \cup \mathcal{V}^{-}(B) .
\end{aligned}
$$

The set $\mathcal{V}_{n s}^{+}(A)$ of propositional variables occurring non-strictly positively in a formula $A$ is defined by

$$
\begin{aligned}
\mathcal{V}_{n s}^{+}(p) & =\mathcal{V}_{n s}^{+}(\perp)=\emptyset, \\
\mathcal{V}_{n s}^{+}(A \wedge B) & =\mathcal{V}_{n s}^{+}(A \vee B)=\mathcal{V}_{n s}^{+}(A) \cup \mathcal{V}_{n s}^{+}(B), \\
\mathcal{V}_{n s}^{+}(A \rightarrow B) & =\mathcal{V}^{-}(A) \cup \mathcal{V}_{n s}^{+}(B)
\end{aligned}
$$

We extend $\mathcal{V}^{+}$to a finite multiset $\Gamma$ of formulas by $\mathcal{V}^{+}(\Gamma)=\bigcup_{A \in \Gamma} \mathcal{V}^{+}(A) . \quad \mathcal{V}^{-}(\Gamma)$ and $\mathcal{V}_{n s}^{+}(\Gamma)$ are defined similarly.

The sequent calculus G3cp is specified by the following axioms and rules:

$$
\begin{array}{cc}
p, \Gamma \Rightarrow \Delta, p \quad \text { Ax } & \perp, \Gamma \Rightarrow \Delta \quad \mathrm{L} \perp \\
\frac{A, B, \Gamma \Rightarrow \Delta}{A \wedge B, \Gamma \Rightarrow \Delta} \mathrm{L} \wedge & \frac{\Gamma \Rightarrow \Delta, A \quad \Gamma \Rightarrow \Delta, B}{\Gamma \Rightarrow \Delta, A \wedge B} \mathrm{R} \wedge \\
\frac{A, \Gamma \Rightarrow \Delta \quad B, \Gamma \Rightarrow \Delta}{A \vee B, \Gamma \Rightarrow \Delta} \mathrm{L} \vee & \frac{\Gamma \Rightarrow \Delta, A, B}{\Gamma \Rightarrow \Delta, A \vee B} \mathrm{R} \vee \\
\frac{\Gamma \Rightarrow \Delta, A \quad B, \Gamma \Rightarrow \Delta}{A \rightarrow B, \Gamma \Rightarrow \Delta} \mathrm{L} \rightarrow & \frac{A, \Gamma \Rightarrow \Delta, B}{\Gamma \Rightarrow \Delta, A \rightarrow B} \mathrm{R} \rightarrow
\end{array}
$$

where in $\mathrm{Ax}, p$ is a propositional variable.

The intuitionistic version G3ip of G3cp has the following form:

$$
\begin{array}{cc}
p, \Gamma \Rightarrow p \quad \text { Ax } & \perp, \Gamma \Rightarrow A \quad \mathrm{~L} \perp \\
\frac{A, B, \Gamma \Rightarrow C}{A \wedge B, \Gamma \Rightarrow C} \mathrm{~L} \wedge & \frac{\Gamma \Rightarrow A \quad \Gamma \Rightarrow B}{\Gamma \Rightarrow A \wedge B} \mathrm{R} \wedge \\
\frac{A, \Gamma \Rightarrow C \quad B, \Gamma \Rightarrow C}{A \vee B, \Gamma \Rightarrow C} \mathrm{~L} \vee & \frac{\Gamma \Rightarrow A}{\Gamma \Rightarrow A \vee B} \mathrm{R} \vee \frac{\Gamma \Rightarrow B}{\Gamma \Rightarrow A \vee B} \mathrm{R} \vee_{2} \\
\frac{A \rightarrow B, \Gamma \Rightarrow A \quad B, \Gamma \Rightarrow C}{A \rightarrow B, \Gamma \Rightarrow C} \mathrm{~L} \rightarrow & \frac{A, \Gamma \Rightarrow B}{\Gamma \Rightarrow A \rightarrow B} \mathrm{R} \rightarrow
\end{array}
$$

where in $\mathrm{Ax}, p$ is a propositional variable. 
Note that having the present sequent calculus formulation (Ax with a propositional variable $p$ instead of a formula $A$ ) allows for an easy treatment of the Basis case in the proof of the main result below.

The structural rules (weakening, contraction and cut) are admissible in G3cp and in G3ip; see [6, 3.4.3,3.4.5,4.1.2]. Those structural rules are formulated in G3ip as follows:

$$
\begin{gathered}
\frac{\Gamma \Rightarrow C}{\Gamma, \Delta \Rightarrow C} \text { LW } \frac{A, A, \Gamma \Rightarrow C}{A, \Gamma \Rightarrow C} \mathrm{LC} \\
\frac{\Gamma \Rightarrow A \quad A, \Gamma^{\prime} \Rightarrow C}{\Gamma, \Gamma^{\prime} \Rightarrow C} \mathrm{Cut}
\end{gathered}
$$

We write $\vdash_{c} \Gamma \Rightarrow \Delta$ and $\vdash_{i} \Gamma \Rightarrow A$ for derivability of sequents $\Gamma \Rightarrow \Delta$ and $\Gamma \Rightarrow A$ in G3cp and in G3ip, respectively.

We introduce the symbol "*" as a special proposition letter (a place holder) and an abbreviation $\neg_{*} A \equiv A \rightarrow *$. It is straightforward to see that if $\vdash_{i} \Gamma \Rightarrow A$ then $\vdash_{i} \Gamma, \neg_{*} A \Rightarrow *$; if $\vdash_{i} \Gamma, \neg_{*} \neg_{*} A \Rightarrow *$ then $\vdash_{i} \Gamma \Rightarrow \neg_{*} A$, and $\vdash_{i} \Gamma, A \Rightarrow *$ if and only if $\vdash_{i} \Gamma \Rightarrow \neg_{*} A$. From the latter and the former results, it is trivial to conclude that if $\vdash_{i} \Gamma, A \Rightarrow *$ then $\vdash_{i} \Gamma, \neg_{*} \neg_{*} A \Rightarrow *$, and $\vdash_{i} \Gamma, \neg_{*} A \Rightarrow *$ if and only if $\vdash_{i} \Gamma \Rightarrow \neg_{*} \neg_{*} A$.

We have the following lemma for the logical operators and the operators $\neg$ and $\neg_{*}$.

\section{Lemma 2.1.}

(1) $\vdash_{i} \Gamma, p \vee \neg p, \neg_{*} \neg p, \neg_{*} p \Rightarrow *$,

(2) $\vdash_{i} \Gamma, \neg_{*} \neg \perp \Rightarrow *$,

(3) $\vdash_{i} \neg_{*} \neg\left(D \wedge D^{\prime}\right) \Rightarrow \neg_{*} \neg D \wedge \neg_{*} \neg D^{\prime}$,

(4) $\vdash_{i} \neg_{*} \neg_{*} S \wedge \neg_{*} \neg_{*} S^{\prime} \Rightarrow \neg_{*} \neg_{*}\left(S \wedge S^{\prime}\right)$,

(5) $\vdash_{i} \neg_{*} \neg\left(D \vee D^{\prime}\right) \Rightarrow \neg_{*} \neg_{*}\left(\neg_{*} \neg D \vee \neg_{*} \neg D^{\prime}\right)$,

(6) $\vdash_{i} \neg_{*}\left(\neg_{*} S \wedge \neg_{*} S^{\prime}\right) \Rightarrow \neg_{*} \neg_{*}\left(S \vee S^{\prime}\right)$,

(7) $\vdash_{i} \neg_{*} \neg(S \rightarrow B) \Rightarrow \neg_{*} \neg_{*} S \rightarrow \neg_{*} \neg B$,

(8) $\vdash_{i} S \rightarrow B \Rightarrow \neg_{*} \neg_{*} S \rightarrow \neg_{*} \neg_{*} B$,

(9) $\vdash_{i} \neg_{*} \neg A \rightarrow \neg_{*} \neg_{*} S \Rightarrow \neg_{*} \neg_{*}(A \rightarrow S)$.

Proof. Easy exercise.

Let $A[* / C]$ denote the result of substituting a formula $C$ for each occurrence of $*$ in a formula $A$, and, for a finite multiset $\Gamma \equiv A_{1}, \ldots, A_{n}$, let $\Gamma[* / C]$ denote the multiset $A_{1}[* / C], \ldots, A_{n}[* / C]$.

Lemma 2.2. If $\vdash_{i} \Gamma \Rightarrow A$, then $\vdash_{i} \Gamma[* / C] \Rightarrow A[* / C]$.

Proof. By induction on the depth of a deduction $\vdash_{i} \Gamma \Rightarrow A$.

\section{THE MAIN RESULT}

If " $c$ " is an operator, such as $\neg$ and $\neg_{*}$, and $\Gamma \equiv A_{1}, \ldots, A_{n}$ is a finite multiset of formulas, then we write $c \Gamma$ for the multiset $c A_{1}, \ldots, c A_{n}$.

Proposition 3.1. If $\vdash_{c} \Gamma, \Delta \Rightarrow \Sigma$, then $\vdash_{i} \Pi_{V}, \Gamma, \neg_{*} \neg \Delta, \neg_{*} \Sigma \Rightarrow *$, where $V$ is a set of propositional variables containing $\left(\mathcal{V}^{-}(\Gamma, \Delta) \cup \mathcal{V}^{+}(\Sigma)\right) \cap\left(\mathcal{V}_{n s}^{+}(\Gamma) \cup \mathcal{V}^{+}(\Delta) \cup \mathcal{V}^{-}(\Sigma)\right)$. 
Proof. Let $V$ be a set of propositional variables containing $\left(\mathcal{V}^{-}(\Gamma, \Delta) \cup \mathcal{V}^{+}(\Sigma)\right) \cap\left(\mathcal{V}_{n s}^{+}(\Gamma) \cup\right.$ $\mathcal{V}^{+}(\Delta) \cup \mathcal{V}^{-}(\Sigma)$ ), and we proceed by induction on the depth of a deduction of $\vdash_{c} \Gamma, \Delta \Rightarrow \Sigma$. Basis. If the deduction is an instance of Ax, then it must be either of the form $p, \Gamma^{\prime}, \Delta \Rightarrow$ $\Sigma^{\prime}, p$, or of the form $\Gamma, p, \Delta^{\prime} \Rightarrow \Sigma^{\prime}, p$. In the former case, we have

$$
\vdash_{i} \Pi_{V}, p, \Gamma^{\prime}, \neg_{*} \neg \Delta, \neg_{*} \Sigma^{\prime}, \neg_{*} p \Rightarrow *
$$

and, in the latter case, since

$$
p \in\left(\mathcal{V}^{-}\left(\Gamma, p, \Delta^{\prime}\right) \cup \mathcal{V}^{+}\left(\Sigma^{\prime}, p\right)\right) \cap\left(\mathcal{V}_{n s}^{+}(\Gamma) \cup \mathcal{V}^{+}\left(p, \Delta^{\prime}\right) \cup \mathcal{V}^{-}\left(\Sigma^{\prime}, p\right)\right) \subseteq V,
$$

we have

$$
\vdash_{i} \Pi_{V}, \Gamma, \neg_{*} \neg p, \neg_{*} \neg \Delta^{\prime}, \neg_{*} \Sigma^{\prime}, \neg_{*} p \Rightarrow *
$$

by Lemma 2.1 (1). If the deduction is an instance of $\mathrm{L} \perp$, then it must be either of the form $\perp, \Gamma^{\prime}, \Delta \Rightarrow \Sigma$, or of the form $\Gamma, \perp, \Delta^{\prime} \Rightarrow \Sigma$. In the former case, we have

$$
\vdash_{i} \Pi_{V}, \perp, \Gamma^{\prime}, \neg_{*} \neg \Delta, \neg_{*} \Sigma \Rightarrow *
$$

and, in the latter case, we have

$$
\vdash_{i} \Pi_{V}, \Gamma, \neg_{*} \neg \perp, \neg_{*} \neg \Delta^{\prime}, \neg_{*} \Sigma \Rightarrow *
$$

by Lemma 2.1 (2).

Induction step. For the induction step, we distinguish the cases: (A) the last rule applied is an L-rule and the principal formula is in $\Delta,(\mathrm{B})$ the last rule applied is an L-rule and the principal formula is in $\Gamma$, and $(\mathrm{C})$ the last rule applied is an R-rule.

Case $A$. The last rule applied is an L-rule, and the principal formula is in $\Delta$.

Case A1. The last rule applied is $\mathrm{L} \wedge$. Then the derivation ends with

$$
\frac{\Gamma, D, D^{\prime}, \Delta^{\prime} \Rightarrow \Sigma}{\Gamma, D \wedge D^{\prime}, \Delta^{\prime} \Rightarrow \Sigma} \mathrm{L} \wedge \text {. }
$$

Since

$$
\begin{aligned}
& \left(\mathcal{V}^{-}\left(\Gamma, D, D^{\prime}, \Delta^{\prime}\right) \cup \mathcal{V}^{+}(\Sigma)\right) \cap\left(\mathcal{V}_{n s}^{+}(\Gamma) \cup \mathcal{V}^{+}\left(D, D^{\prime}, \Delta^{\prime}\right) \cup \mathcal{V}^{-}(\Sigma)\right)= \\
& \quad\left(\mathcal{V}^{-}\left(\Gamma, D \wedge D^{\prime}, \Delta^{\prime}\right) \cup \mathcal{V}^{+}(\Sigma)\right) \cap\left(\mathcal{V}_{n s}^{+}(\Gamma) \cup \mathcal{V}^{+}\left(D \wedge D^{\prime}, \Delta^{\prime}\right) \cup \mathcal{V}^{-}(\Sigma)\right) \subseteq V
\end{aligned}
$$

we have

$$
\vdash_{i} \Pi_{V}, \Gamma, \neg_{*} \neg D, \neg_{*} \neg D^{\prime}, \neg_{*} \neg \Delta^{\prime}, \neg_{*} \Sigma \Rightarrow *
$$

by the induction hypothesis, and hence

$$
\vdash_{i} \Pi_{V}, \Gamma, \neg_{*} \neg D \wedge \neg_{*} \neg D^{\prime}, \neg * \neg \Delta^{\prime}, \neg_{*} \Sigma \Rightarrow *
$$

by L^. Therefore $\vdash_{i} \Pi_{V}, \Gamma, \neg_{*} \neg\left(D \wedge D^{\prime}\right), \neg_{*} \neg \Delta^{\prime}, \neg_{*} \Sigma \Rightarrow *$, by Cut with Lemma 2.1 (3).

Case A2. The last rule applied is $\mathrm{L} \vee$. Then the derivation ends with

$$
\frac{\Gamma, D, \Delta^{\prime} \Rightarrow \Sigma \quad \Gamma, D^{\prime}, \Delta^{\prime} \Rightarrow \Sigma}{\Gamma, D \vee D^{\prime}, \Delta^{\prime} \Rightarrow \Sigma} \mathrm{LV} \text {. }
$$

Since $\left(\mathcal{V}^{-}\left(\Gamma, D, \Delta^{\prime}\right) \cup \mathcal{V}^{+}(\Sigma)\right) \cap\left(\mathcal{V}_{n s}^{+}(\Gamma) \cup \mathcal{V}^{+}\left(D, \Delta^{\prime}\right) \cup \mathcal{V}^{-}(\Sigma)\right) \subseteq V$ and $\left(\mathcal{V}^{-}\left(\Gamma, D^{\prime}, \Delta^{\prime}\right) \cup\right.$ $\left.\mathcal{V}^{+}(\Sigma)\right) \cap\left(\mathcal{V}_{n s}^{+}(\Gamma) \cup \mathcal{V}^{+}\left(D^{\prime}, \Delta^{\prime}\right) \cup \mathcal{V}^{-}(\Sigma)\right) \subseteq V$, we have

$$
\vdash_{i} \Pi_{V}, \Gamma, \neg_{*} \neg D, \neg_{*} \neg \Delta^{\prime}, \neg_{*} \Sigma \Rightarrow * \quad \text { and } \quad \vdash_{i} \Pi_{V}, \Gamma, \neg_{*} \neg D^{\prime}, \neg_{*} \neg \Delta^{\prime}, \neg_{*} \Sigma \Rightarrow *
$$

by the induction hypothesis, and hence

$$
\vdash_{i} \Pi_{V}, \Gamma, \neg_{*} \neg D \vee \neg_{*} \neg D^{\prime}, \neg_{*} \neg \Delta^{\prime}, \neg_{*} \Sigma \Rightarrow *
$$


by $L \vee$. Therefore

$$
\vdash_{i} \Pi_{V}, \Gamma, \neg_{*} \neg_{*}\left(\neg_{*} \neg D \vee \neg * \neg D^{\prime}\right), \neg_{*} \neg \Delta^{\prime}, \neg_{*} \Sigma \Rightarrow *
$$

and so $\vdash_{i} \Pi_{V}, \Gamma, \neg_{*} \neg\left(D \vee D^{\prime}\right), \neg_{*} \neg \Delta^{\prime}, \neg_{*} \Sigma \Rightarrow *$, by Cut with Lemma 2.1 (5).

Case A3. The last rule applied is $\mathrm{L} \rightarrow$. Then the derivation ends with

$$
\frac{\Gamma, \Delta^{\prime} \Rightarrow \Sigma, S \quad B, \Gamma, \Delta^{\prime} \Rightarrow \Sigma}{\Gamma, S \rightarrow B, \Delta^{\prime} \Rightarrow \Sigma} \mathrm{L} \rightarrow
$$

Since

$$
\begin{aligned}
& \left(\mathcal{V}^{-}\left(\Gamma, \Delta^{\prime}\right) \cup \mathcal{V}^{+}(\Sigma, S)\right) \cap\left(\mathcal{V}_{n s}^{+}(\Gamma) \cup \mathcal{V}^{+}\left(\Delta^{\prime}\right) \cup \mathcal{V}^{-}(\Sigma, S)\right) \subseteq \\
& \quad\left(\mathcal{V}^{-}\left(\Gamma, S \rightarrow B, \Delta^{\prime}\right) \cup \mathcal{V}^{+}(\Sigma)\right) \cap\left(\mathcal{V}_{n s}^{+}(\Gamma) \cup \mathcal{V}^{+}\left(S \rightarrow B, \Delta^{\prime}\right) \cup \mathcal{V}^{-}(\Sigma)\right) \subseteq V
\end{aligned}
$$

and

$$
\begin{aligned}
& \left(\mathcal{V}^{-}\left(\Gamma, B, \Delta^{\prime}\right) \cup \mathcal{V}^{+}(\Sigma)\right) \cap\left(\mathcal{V}_{n s}^{+}(\Gamma) \cup \mathcal{V}^{+}\left(B, \Delta^{\prime}\right) \cup \mathcal{V}^{-}(\Sigma)\right) \subseteq \\
& \quad\left(\mathcal{V}^{-}\left(\Gamma, S \rightarrow B, \Delta^{\prime}\right) \cup \mathcal{V}^{+}(\Sigma)\right) \cap\left(\mathcal{V}_{n s}^{+}(\Gamma) \cup \mathcal{V}^{+}\left(S \rightarrow B, \Delta^{\prime}\right) \cup \mathcal{V}^{-}(\Sigma)\right) \subseteq V
\end{aligned}
$$

we have

$$
\vdash_{i} \Pi_{V}, \Gamma, \neg_{*} \neg \Delta^{\prime}, \neg_{*} \Sigma, \neg_{*} S \Rightarrow * \quad \text { and } \quad \vdash_{i} \Pi_{V}, \Gamma, \neg_{*} \neg B, \neg_{*} \neg \Delta^{\prime}, \neg_{*} \Sigma \Rightarrow *
$$

by the induction hypothesis, and therefore, since

$$
\vdash_{i} \Pi_{V}, \Gamma, \neg_{*} \neg \Delta^{\prime}, \neg_{*} \Sigma \Rightarrow \neg_{*} \neg_{*} S
$$

we have $\vdash_{i} \Pi_{V}, \Gamma, \neg_{*} \neg_{*} S \rightarrow \neg_{*} \neg B, \neg_{*} \neg \Delta^{\prime}, \neg_{*} \Sigma \Rightarrow \neg_{*} \neg_{*} S$, by LW. Thus

$$
\vdash_{i} \Pi_{V}, \Gamma, \neg_{*} \neg_{*} S \rightarrow \neg_{*} \neg B, \neg_{*} \neg \Delta^{\prime}, \neg_{*} \Sigma \Rightarrow *
$$

by $\mathrm{L} \rightarrow$, and so $\vdash_{i} \Pi_{V}, \Gamma, \neg_{*} \neg(S \rightarrow B), \neg_{*} \neg \Delta^{\prime}, \neg_{*} \Sigma \Rightarrow *$, by Cut with Lemma 2.1 (17).

Case $B$. The last rule applied is an L-rule, and the principal formula is in $\Gamma$. Since the cases for the rules $L \wedge$ and $L \vee$ are straightforward, we review the case for the rule $L \rightarrow$.

Case B1. The last rule applied is $\mathrm{L} \rightarrow$. Then the derivation ends with

$$
\frac{\Gamma^{\prime}, \Delta \Rightarrow \Sigma, S \quad B, \Gamma^{\prime}, \Delta \Rightarrow \Sigma}{S \rightarrow B, \Gamma^{\prime}, \Delta \Rightarrow \Sigma} \mathrm{L} \rightarrow
$$

Since

$$
\begin{aligned}
& \left(\mathcal{V}^{-}\left(\Gamma^{\prime}, \Delta\right) \cup \mathcal{V}^{+}(\Sigma, S)\right) \cap\left(\mathcal{V}_{n s}^{+}\left(\Gamma^{\prime}\right) \cup \mathcal{V}^{+}(\Delta) \cup \mathcal{V}^{-}(\Sigma, S)\right) \subseteq \\
& \quad\left(\mathcal{V}^{-}\left(S \rightarrow B, \Gamma^{\prime}, \Delta\right) \cup \mathcal{V}^{+}(\Sigma)\right) \cap\left(\mathcal{V}_{n s}^{+}\left(S \rightarrow B, \Gamma^{\prime}\right) \cup \mathcal{V}^{+}(\Delta) \cup \mathcal{V}^{-}(\Sigma)\right) \subseteq V
\end{aligned}
$$

and

$$
\begin{aligned}
& \left(\mathcal{V}^{-}\left(B, \Gamma^{\prime}, \Delta\right) \cup \mathcal{V}^{+}(\Sigma)\right) \cap\left(\mathcal{V}_{n s}^{+}\left(B, \Gamma^{\prime}\right) \cup \mathcal{V}^{+}(\Delta) \cup \mathcal{V}^{-}(\Sigma)\right) \subseteq \\
& \quad\left(\mathcal{V}^{-}\left(S \rightarrow B, \Gamma^{\prime}, \Delta\right) \cup \mathcal{V}^{+}(\Sigma)\right) \cap\left(\mathcal{V}_{n s}^{+}\left(S \rightarrow B, \Gamma^{\prime}\right) \cup \mathcal{V}^{+}(\Delta) \cup \mathcal{V}^{-}(\Sigma)\right) \subseteq V,
\end{aligned}
$$

we have

$$
\vdash_{i} \Pi_{V}, \Gamma^{\prime}, \neg_{*} \neg \Delta, \neg_{*} \Sigma, \neg_{*} S \Rightarrow * \quad \text { and } \quad \vdash_{i} \Pi_{V}, B, \Gamma^{\prime}, \neg_{*} \neg \Delta, \neg_{*} \Sigma \Rightarrow *
$$

by the induction hypothesis, and therefore, since

$$
\vdash_{i} \Pi_{V}, \Gamma^{\prime}, \neg_{*} \neg \Delta, \neg_{*} \Sigma \Rightarrow \neg_{*} \neg_{*} S
$$

we have $\vdash_{i} \Pi_{V}, \neg_{*} \neg_{*} S \rightarrow \neg_{*} \neg_{*} B, \Gamma^{\prime}, \neg_{*} \neg \Delta, \neg_{*} \Sigma \Rightarrow \neg_{*} \neg_{*} S$, by LW, and

$$
\vdash_{i} \Pi_{V}, \neg_{*} \neg_{*} B, \Gamma^{\prime}, \neg_{*} \neg \Delta, \neg_{*} \Sigma \Rightarrow * \text {. }
$$


Thus

$$
\vdash_{i} \Pi_{V}, \neg_{*} \neg_{*} S \rightarrow \neg_{*} \neg_{*} B, \Gamma^{\prime}, \neg_{*} \neg \Delta, \neg_{*} \Sigma \Rightarrow *
$$

by $\mathrm{L} \rightarrow$, and so $\vdash_{i} \Pi_{V}, S \rightarrow B, \Gamma^{\prime}, \neg_{*} \neg \Delta, \neg_{*} \Sigma \Rightarrow *$, by Cut with Lemma 2.1 (8).

Case $C$. The last rule applied is an R-rule.

Case $C 1$. The last rule applied is $\mathrm{R} \wedge$. Then the derivation ends with

$$
\frac{\Gamma, \Delta \Rightarrow \Sigma^{\prime}, S \quad \Gamma, \Delta \Rightarrow \Sigma^{\prime}, S^{\prime}}{\Gamma, \Delta \Rightarrow \Sigma^{\prime}, S \wedge S^{\prime}} \mathrm{R} \wedge \text {. }
$$

Since $\left(\mathcal{V}^{-}(\Gamma, \Delta) \cup \mathcal{V}^{+}\left(\Sigma^{\prime}, S\right)\right) \cap\left(\mathcal{V}_{n s}^{+}(\Gamma) \cup \mathcal{V}^{+}(\Delta) \cup \mathcal{V}^{-}\left(\Sigma^{\prime}, S\right)\right) \subseteq V$ and $\left(\mathcal{V}^{-}(\Gamma, \Delta) \cup \mathcal{V}^{+}\left(\Sigma^{\prime}, S^{\prime}\right)\right) \cap$ $\left(\mathcal{V}_{n s}^{+}(\Gamma) \cup \mathcal{V}^{+}(\Delta) \cup \mathcal{V}^{-}\left(\Sigma^{\prime}, S^{\prime}\right)\right) \subseteq V$, we have

$$
\vdash_{i} \Pi_{V}, \Gamma, \neg_{*} \neg \Delta, \neg_{*} \Sigma^{\prime}, \neg_{*} S \Rightarrow * \quad \text { and } \quad \vdash_{i} \Pi_{V}, \Gamma, \neg_{*} \neg \Delta, \neg_{*} \Sigma^{\prime}, \neg_{*} S^{\prime} \Rightarrow *
$$

by the induction hypothesis, and hence

$$
\vdash_{i} \Pi_{V}, \Gamma, \neg_{*} \neg \Delta, \neg_{*} \Sigma^{\prime} \Rightarrow \neg_{*} \neg_{*} S \quad \text { and } \quad \vdash_{i} \Pi_{V}, \Gamma, \neg_{*} \neg \Delta, \neg_{*} \Sigma^{\prime} \Rightarrow \neg_{*} \neg_{*} S^{\prime} .
$$

Therefore $\vdash_{i} \Pi_{V}, \Gamma, \neg_{*} \neg \Delta, \neg_{*} \Sigma^{\prime} \Rightarrow \neg_{*} \neg_{*}\left(S \wedge S^{\prime}\right)$, by R^ and Cut with Lemma 2.1 (4), and so $\vdash_{i} \Pi_{V}, \Gamma, \neg_{*} \neg \Delta, \neg_{*} \Sigma^{\prime}, \neg_{*}\left(S \wedge S^{\prime}\right) \Rightarrow *$.

Case C2. The last rule applied is $\mathrm{R} \vee$. Then the derivation ends with

$$
\frac{\Gamma, \Delta \Rightarrow \Sigma^{\prime}, S, S^{\prime}}{\Gamma, \Delta \Rightarrow \Sigma^{\prime}, S \vee S^{\prime}} \mathrm{R} \vee
$$

Since $\left(\mathcal{V}^{-}(\Gamma, \Delta) \cup \mathcal{V}^{+}\left(\Sigma^{\prime}, S, S^{\prime}\right)\right) \cap\left(\mathcal{V}_{n s}^{+}(\Gamma) \cup \mathcal{V}^{+}(\Delta) \cup \mathcal{V}^{-}\left(\Sigma^{\prime}, S, S^{\prime}\right)\right) \subseteq V$, we have

$$
\vdash_{i} \Pi_{V}, \Gamma, \neg_{*} \neg \Delta, \neg_{*} \Sigma^{\prime}, \neg_{*} S, \neg_{*} S^{\prime} \Rightarrow *
$$

by the induction hypothesis, and hence

$$
\vdash_{i} \Pi_{V}, \Gamma, \neg_{*} \neg \Delta, \neg_{*} \Sigma^{\prime}, \neg_{*} S \wedge \neg_{*} S^{\prime} \Rightarrow *
$$

by L^. Therefore $\vdash_{i} \Pi_{V}, \Gamma, \neg_{*} \neg \Delta, \neg_{*} \Sigma^{\prime} \Rightarrow \neg_{*}\left(\neg_{*} S \wedge \neg_{*} S^{\prime}\right)$, and so

$$
\vdash_{i} \Pi_{V}, \Gamma, \neg_{*} \neg \Delta, \neg_{*} \Sigma^{\prime} \Rightarrow \neg_{*} \neg_{*}\left(S \vee S^{\prime}\right)
$$

by Cut with Lemma 2.1 (6). Thus $\vdash_{i} \Pi_{V}, \Gamma, \neg_{*} \neg \Delta, \neg_{*} \Sigma^{\prime}, \neg_{*}\left(S \vee S^{\prime}\right) \Rightarrow *$.

Case C3. The last rule applied is $\mathrm{R} \rightarrow$. Then the derivation ends with

$$
\frac{A, \Gamma, \Delta \Rightarrow \Sigma^{\prime}, S}{\Gamma, \Delta \Rightarrow \Sigma^{\prime}, A \rightarrow S} \mathrm{R} \rightarrow
$$

Since

$$
\begin{aligned}
& \left(\mathcal{V}^{-}(\Gamma, A, \Delta) \cup \mathcal{V}^{+}\left(\Sigma^{\prime}, S\right)\right) \cap\left(\mathcal{V}_{n s}^{+}(\Gamma) \cup \mathcal{V}^{+}(A, \Delta) \cup \mathcal{V}^{-}\left(\Sigma^{\prime}, S\right)\right)= \\
& \quad\left(\mathcal{V}^{-}(\Gamma, \Delta) \cup \mathcal{V}^{+}\left(\Sigma^{\prime}, A \rightarrow S\right)\right) \cap\left(\mathcal{V}_{n s}^{+}(\Gamma) \cup \mathcal{V}^{+}(\Delta) \cup \mathcal{V}^{-}\left(\Sigma^{\prime}, A \rightarrow S\right)\right) \subseteq V
\end{aligned}
$$

we have

$$
\vdash_{i} \Pi_{V}, \Gamma, \neg_{*} \neg A, \neg_{*} \neg \Delta, \neg_{*} \Sigma^{\prime}, \neg_{*} S \Rightarrow *
$$

by the induction hypothesis, and therefore, since

$$
\vdash_{i} \Pi_{V}, \Gamma, \neg_{*} \neg A, \neg_{*} \neg \Delta, \neg_{*} \Sigma^{\prime} \Rightarrow \neg_{*} \neg_{*} S
$$

we have $\vdash_{i} \Pi_{V}, \Gamma, \neg_{*} \neg \Delta, \neg_{*} \Sigma^{\prime} \Rightarrow \neg_{*} \neg A \rightarrow \neg_{*} \neg_{*} S$, by $\mathrm{R} \rightarrow$. Thus

$$
\vdash_{i} \Pi_{V}, \Gamma, \neg_{*} \neg \Delta, \neg_{*} \Sigma^{\prime} \Rightarrow \neg_{*} \neg_{*}(A \rightarrow S)
$$

by Cut with Lemma 2.1 (9), and so $\vdash_{i} \Pi_{V}, \Gamma, \neg_{*} \neg \Delta, \neg_{*} \Sigma^{\prime}, \neg_{*}(A \rightarrow S) \Rightarrow *$. 
Theorem 3.2. If $\vdash_{c} \Gamma \Rightarrow A$, then $\vdash_{i} \Pi_{V}, \Gamma \Rightarrow A$, where $V=\left(\mathcal{V}^{-}(\Gamma) \cup \mathcal{V}^{+}(A)\right) \cap\left(\mathcal{V}_{n s}^{+}(\Gamma) \cup\right.$ $\left.\mathcal{V}^{-}(A)\right)$.

Proof. Suppose that $\vdash_{c} \Gamma \Rightarrow A$, and let $V=\left(\mathcal{V}^{-}(\Gamma) \cup \mathcal{V}^{+}(A)\right) \cap\left(\mathcal{V}_{n s}^{+}(\Gamma) \cup \mathcal{V}^{-}(A)\right)$. Then $\vdash_{i} \Pi_{V}, \Gamma, \neg_{*} A \Rightarrow *$, by Proposition [3.1, and hence

$$
\vdash_{i} \Pi_{V}, \Gamma, A \rightarrow A \Rightarrow A
$$

by Lemma 2.2. Therefore $\vdash_{i} \Pi_{V}, \Gamma \Rightarrow A$.

Corollary 3.3. If $\vdash_{c} \Gamma \Rightarrow A$ and $\left(\mathcal{V}^{-}(\Gamma) \cup \mathcal{V}^{+}(A)\right) \cap\left(\mathcal{V}_{n s}^{+}(\Gamma) \cup \mathcal{V}^{-}(A)\right)=\emptyset$, then $\vdash_{i} \Gamma \Rightarrow A$.

\section{ACKNowledgement}

The author thanks the Japan Society for the Promotion of Science (Grant-in-Aid for Scientific Research (C) No.23540130) for partly supporting the research.

\section{REFERENCES}

[1] Ken-etsu Fujita, $\mu$-head form proofs with at most two formulas in the succedent, Trans. Inform. Process. Soc. Japan, 38 (1997), 1073-1082.

[2] Hajime Ishihara, A note on the Gödel-Gentzen translation, MLQ Math. Log. Q. 46 (2000), 135-137.

[3] Hajime Ishihara, Some conservative extension results on classical and intuitionistic sequent calculi, In: U. Berger, H. Diener, P. Schuster and M. Seisenberger eds., Logic, Construction, Computation, Ontos Verlag, Frankfurt, 2012, 289-304.

[4] Sara Negri and Jan von Plato, Structural Proof Theory, Cambridge University Press, Cambridge, 2001.

[5] Anne S. Troelstra and Dirk van Dalen, Constructivism in Mathematics, Vol. I and II, North-Holland, Amsterdam, 1988.

[6] Anne S. Troelstra and Helmut Schwichtenberg, Basic Proof Theory, Cambridge Tracts in Theoretical Computer Science, 43, Cambridge University Press, Cambridge, 1996.

[7] Jan von Plato, Proof theory of full classical propositional logic, preprint, 1998. 